\title{
LA VIOLENCIA INTRAFAMILIAR, EL USO DE DROGAS EN LA PAREJA, DESDE LA PERSPECTIVA DE LA MUJ ER MALTRATADA ${ }^{1}$
}

Rosa G. Vaiz Bonifaz Ana Marcia Spanó Nakano

Bonifaz RGV, Nakano AMS. La violencia intrafamiliar, el uso de drogas en la pareja, desde la perspectiva de la mujer maltratada. Rev Latino-am Enfermagem 2004 março-abril; 12(número especial):433-8.

El presente estudio tiene la finalidad de visibilizar la violencia que afecta a las mujeres, teniendo como principales elementos precipitantes de la actitud violenta del agresor la ingesta de alcohol y/o drogas. Los objetivos del presente estudio son: conocer los significados que la mujer le atribuye a la violencia; reconocer los diferentes factores relacionados a la ocurrencia de la violencia intrafamiliar e identificar como se procesa el consumo de drogas en la pareja. El tipo de estudio es exploratorio y descriptivo, se utilizó la metodología cualitativa, el recorte empírico lo constituyeron seis mujeres, quienes acudieron a realizar su denuncia a la Comisaria de Mujeres de Lima. Los hallazgos muestran que el tiempo de unión osciló entre dos meses y dieciocho años, ocurriendo el maltrato en casi todo el tiempo de unión. Con respecto al tipo de violencia sufrida la de mayor magnitud fue la física; de forma más sutil la psicológica, la violencia sexual no fue referida, el dinero y celos fueron referidos como focos desencadenantes de las discusiones, las que están asociados al uso de alcohol y droga en el agresor. Las categorías establecidas fueron: el tipo de violencia sufrida por las mujeres, el contexto de la violencia, la repercusión de la violencia sufrida por la mujer en su salud y la reacción de violencia sufrida por medio de la denuncia. Como principales conclusiones tenemos que la violencia contra las mujeres se revela como tema social y sanitario, con repercusiones en la morbi-mortalitad y en términos de calidad de vida de las mujeres., en el campo de la salud, es necesario reconocer a las mujeres en situación de violencia, considerar como factores de riesgo para la pareja el uso de alcohol y drogas, las mujeres ocultan por vergüenza la problemática que viven, el personal de salud debe ser capaz de diagnosticar la violencia en sus diferentes formas, expresadas por quejas o dolores crónicos sin causas aparentes.

DESCRIPTORES: violencia; familia; enfermería; preparaciones farmacéuticas

\section{THE VIOLENCE INTRAFAMILIAR, THE USE OF DRUGS IN THE COUPLE, FROM THE MISTREATED WOMAN'S PERSPECTIVE}

The present study has the visibilizar purpose the violence that affects the women, having as main elements precipitants of the aggressor's violent attitude the ingest of alcohol and/or it drugs. The objectives of the present study are: to know the meanings that the woman attributes to the violence; to recognize the different factors related to the occurrence of the family violence and to identify like the consumption of drugs is processed in the couple. The study type is exploratory and descriptive, the qualitative methodology, the empiric cutting was used six women they constituted it who went to carry out its accusation to the Police station of Women from Lima. The discoveries show that the time of union oscillated between two months and eighteen years, happening the abuse in case the whole time of union. With regard to the type of suffering violence that of more magnitude was the physics; in a subtler way the psychological one, the sexual violence was not referred, the money and jealousies were referred as focuses desencadenantes of the discussions, those that are associated to the use of alcohol and it drugs in the aggressor. The established categories were: the type of violence undergone by the women, the context of the violence, the repercussion of the violence undergone by the women in its health and the reaction of violence undergone by means of the denunciation. As main conclusions we have that the violence against the women is revealed like social and sanitary topic, with repercussions in the morbimortality and in terms of warm of the women's life, in the field of the health, it is necessary to recognize the women in situations of violence, to consider as factors of risk for the couple the use of the alcohol and drugs, the women hide for shame the problem that you/they live, the personnel of health it should be able to diagnose the violence in their different forms, expressed by complaints or chronic pains without apparent causes.

DESCRIPTORS: violence; family; nursing; pharmacentical preparations

\section{A VIOLENCIA INTRAFAMILIAR, O USO DA DROGA NO CASAL, DESDE A} PERSPECTIVA DA MULHER MALTRATADA

O presente estudio tem a finalidade de fazer visivel a violência que afeita as mulheres, tendo como elementos principais a atitude violenta do agresor por ingesta de álcool e drogas. Os objetivos do presente estudo são conhecer os significados que a mulher atribui a violência; reconhecer os diferentes fatores relacionados á ocorrência da violência intrafamiliar e identificar como é o processo de consumo de drogas no casal. O tipo de estudo é exploratorio e descritivo, foi utilizada a metodologia qualitativa, o recorte empírico o constituiu seis mulheres que realizam a denuncia na Comisaria de mulheres de Lima. Os hallazgos mostram que o tempo deunião oscilo entre dois meses e dezoito anos, acontecendo o maltrato em quasi o tempo todo da uniao. Com respeito ao tipo de violência sofrida a de maior magnitude foi a violencia fisica, a violência psicologica foi menor e a violência sexual nao foi referida. $O$ dineiro e a siume foram referidos como foucos desencadenantes das discussões, as que estao asociadas ao uso de álcool e droga no agressor. As categorias emergentes foram: $O$ tipo de violência sofrida pelas mulheres, o contexto da violência, a repercusao da violência sofrida pela mulher na sua saude e a reação da violência sofrida por meio do reclamo. As principais conclusões que temos é que a violência contra as mulheres é evidenciada como um tema social e sanitario, com repercusoes na morbi-mortalidade e qualidade de vida das mulheres, no campo da saude, é necesario reconhecer ás mulheres em situação de violência, considerar como fatores de risco para o casal o consumo de álcool e drogas, as mulheres ocultam por vergonha a problemática que vivem, o pessoal da saude é capaz de diagnosticar a violência em seus diferentes formas, expresadas por queixas e a dor crônica sem causas aparentes.

DESCRITORES: violência; família; enfermagem; preparações farmacêuticas

\footnotetext{
${ }^{1}$ Las opiniones expresadas en este articulo son de responsabilidad exclusiva de los autores y no representan la posición de la organización donde trabajan o de su administración; ${ }^{2}$ Licenciada en Enfermería, Mester en Enfermería, Docente de la Facultad de Enfermería de la Universidad Peruana Cayetano Heredia, correo electrónico: rvaiz@upch.edu.pe; ${ }^{3}$ Docente de la Escuela de Enfermería de Ribeirão Preto, de la Universidad de São Paulo, Centro Colaborador de la OMS para el desarrollo de la investigación en enfermería, correo electrónico: nakano@eerp.usp.br
} 
INTRODUCCIÓN

La violencia afecta a las mujeres independientemente de la cultura, clase social, etnia, educación y orientación sexual. Estudios realizados revelan que a nivel mundial, al menos el $25 \%$ de las mujeres han sido abusadas.

El concepto de violencia familiar que se referirá en el presente estudio será el mencionado por la Organización Mundial de la Salud ${ }^{(1)}$ que la define como "todo acto de violencia basado en el género que tiene como resultado posible o real un daño físico, sexual o psicológico, incluidas las amenazas la coerción o la privación arbitraria de la libertad, ya sea que ocurra en la vida pública o en la vida privada.

Observa la autora que los principales elementos precipitantes de la actitud violenta del agresor se relacionan con la ingesta de alcohol y/o drogas, aunque también pueden responder a otras múltiples causalidades psicológicas y sociales. En consecuencia, el uso indebido de drogas se constituye en "factor desencadenante" cuando propicia violencia en el ámbito familiar, o en "efecto" en tanto las drogas se convierten en refugio o escape de una persona para sobrellevar situaciones de violencia familiar $^{(2)}$.

El abuso de alcohol y de drogas son fenómenos que se incrementan en los últimos años. En la región de las América ese incremento se observa en forma alarmante y el Perú no está exento de esta problemática, tal como lo demuestra el estudio epidemiológico realizado por el control de abuso de Drogas en el Perú por CONTRADROGAS en el año1999.

Con relación a la prevalencia de violencia intra familiar, el Programa de Naciones Unidas para el desarrollo (PNUD) emitió el informe Perú, para señalar que las denuncias recepcionadas entre 1994 y 1997 en Lima suman alrededor de 20515, para 1997 el número de denuncias recibidas por la Primera Comisaría de Mujeres en Lima fue de alrededor de 6000 lo que revela un significativo incremento de más de un $300 \%$ con respecto al año anterior.

Sólo en 1997, del total de denuncias registradas en Lima, el 76.5\% fueron por violencia física, es decir 18,801 registradas en Lima, el 76.5\% fueron por violencia física, es decir 18,801 y 5,775 fueron por violencia psicológica, lo que representa un $23.5 \%$. Así mismo, el $74.4 \%$ de los agresores se encontraban ecuánimes y el $24.1 \%$ en estado de ebriedad y $1.5 \%$ drogadas $^{(3)}$.
Estos problemas sociales motivan nuestro interés de estudio, ya que en la labor diaria como enfermera, se observa que las mujeres acuden a los servicios de salud por presentar síntomas de afecciones físicas, emocionales que pueden surgir como consecuencia de vivir en clima de violencia de género con su pareja, sea esposo o conviviente. Solicitan entonces atención que puede ser los servicios de emergencia por presentar lesiones agudas (abortos, heridas, contusiones, fracturas, intento de suicidio), y la causa se refleja claramente o algunas veces la agredida enmascara el origen de las lesiones ${ }^{(4)}$.

Otras mujeres por el impacto de la violencia tienden a presentar molestias crónicas (como la migraña, anemia, ansiedad, depresión, dolores crónicos, etc.) y al momento que solicita atención no refieren vivir en ambiente de violencia $^{(5)}$

\section{MÉTODOS}

El presente estudio se realizó, a través de la metodología cualitativa. El universo empírico lo constituyeron las mujeres que acudieron a realizar denuncia de violencia intra familiar, de parte de su pareja, a la Comisaría de Mujeres de Lima.

En Lima Metropolitana existen siete comisarias especializadas, siendo la de Lima-Cercado, la primera en crearse en el año 1988, las restantes están ubicadas en los distritos se San Juan de Lurigancho, Villa EI Salvador, Collique, Callao, Independencia y en el Ministerio de la Mujer y Desarrollo Social. Los servicios que brindan son de asesoría legal, peritaje y orientación psicológica, servicio social, coordinación con medicina legal : dependencia del Ministerio del Interior.

La colecta de datos entre los meses de Agosto a Setiembre del 2002, e tamaño de muestra se estableció mediante el criterio de saturación ${ }^{(6)}$. El análisis de datos es realizado en base a la técnica de análisis de contenido.

\section{OBJETIVOS}

Los objetivos del estudio son identificar el significado que la mujer le atribuye a la violencia sufrida, así como reconocer los diferentes factores relacionados a la ocurrencia de la violencia intra familiar e identificar como se procesa el consumo de drogas en la pareja en el contexto de la violencia intrafamiliar. 


\section{HALLAZGOS Y DISCUSIÓN}

Perfil de las mujeres

Trazando un perfil de las 06 mujeres entrevistadas en nuestro estudio, las edades varían de 22 a 41 años. Tales datos son compatibles a las halladas en el estudio de la Organización Mundial de la Salud sobre violencia sexual y física contra las mujeres en el Perú donde encontraron que más de la mitad de las mujeres de Lima han sido agredidas física o sexualmente por su pareja, se precisa que se trata del $51 \%$ de la población femenina limeña de 15 a 49 años $^{(1)}$.

En el Cusco donde también investigó por primera vez la OMS, las víctimas se elevan al $68 \%$, en la población también de 15 a 49 años ${ }^{(7)}$.

En relación al estado civil de las entrevistadas fueron 02 casadas, 04 convivientes. El tiempo de convivencia, se encontró que fue de 02 meses a 18 años y en relación al tiempo de maltrato, este fue casi todo el tiempo que vivieron juntos y en un caso prácticamente como ex - conviviente.

La tolerancia de las mujeres podrá ser explicada que las víctimas de la violencia consideran que los problemas de la pareja se deben resolver en el hogar e incluso tres de cada cuatro cusqueñas consideran que solo deben obedecer al esposo o pareja ${ }^{(7)}$.

Las relaciones de género, a pesar de los cambios relevantes ocurridos en las últimas décadas, aún prevalecen ideas de jerarquías entre hombres y mujeres. El proceso de construcción simbólica y cultural de lo que es masculino y femenino está reservado a la mujer, en la mayoría de las veces, la condición de víctima y, al hombre el de agresor potencial o efectivo. El hombre es aún identificado como protector de la mujer, frente a otros hombres.

En el rol ocupacional de las mujeres del estudio fueron de amas de casa, ocupación técnica como costurera, peinadora, dibujante. En relación a los varones se desempeñaban como comerciantes, obreros, técnicos dental y alguno sin ocupación.

Tradicionalmente, el hombre ejerce su autoridad, garantizando los recursos materiales, el respeto y la protección de la familia. Son construcciones sociales que colocan a la mujer en una condición de vulnerabilidad, en la medida que su relación con el mundo externo o público, de poder político y económico, es mediado por el hombre fragilizando frente a este mundo que, a su vez, reproduce y reitera las diferencias sexuales. De esta condición de género es que resulta para el trabajo femenino de carácter de complementación de la renta familiar, de baja remuneración, posición secundaria y discriminada en el mercado de trabajo.

El tipo de violencia sufrida por las mujeres estudiadas

Las mujeres son víctimas, principalmente, de las llamadas violencias físicas, psicológicas y sexual. Lo que se puede desprender de las entrevistas es que el tipo de violencia de mayor magnitud es la física, en sus diversas dimensiones, y sexual que se refleja en las siguientes manifestaciones:

...Golpes en el cuerpo, intento de ahorcamiento, electrocución, abuso sexual (E.1)

...Maltrato físico y psicológico ...me golpea en mi cuerpo y en la cabeza con palo (E.4)

...Sólo tres meses que vivo con él y tres veces me ha maltratado física y verbalmente...intentando ahorcarme (E.5)

...Me dio puntapiés en mi barriga, ahora me duele mucho...está grávida de dos meses (E.5)

Otro aspecto que observamos en el grupo, es que dos de las víctimas estaban embarazadas, una de ellas refirió agresiones directas en el vientre. A ese respecto Mc Farlene, considera que la violencia doméstica es más probable que ocurra durante la gestación, ocasionando daños a la salud de la mujer y al niño ${ }^{(8)}$.

También están presentes formas más sutiles, las agresiones psicológica, los testimonios:

...Amenaza de aislamiento (E.1)

...Me insulta, me dice cosas fuertes...delante de cualquier persona, me trata mal, me falta el respeto (E.3)

...Amenaza a cualquier hora, puede ser incluso en la madrugada (E.4)

...Quiere que me vaya de la casa, dice que no tengo derecho (la casa fue pagada en parte con el dinero de mi difunto marido) (E.4)

Esa forma de violencia no deja marcas en el en el cuerpo, mas causa impacto profundo llevando a la destrucción emocional, afectando la vida de la mujer. La violencia psicológica* es toda acción u omisión que causa

\footnotetext{
* Definición de violencia física, psicológica y sexual - Brasil, Ministério de Salud. Acciones Institucionales, Promoviendo la Salud de la Mujer. Revista Promoción de la Salud, año 3, n 6, Octubre 2002
} 
daño a la autoestima, a la identidad o al desenvolvimiento de la persona. En la práctica, se presenta como formas de amenazas, humillaciones, chantajes críticas, reclamo de comportamientos, a través del lenguaje verbal, gesticular y comportamental, que ocurre de forma disimulada o explícita, tornándose la víctima sumisa al agresor y, muchas veces, llegando a considerársele culpable de las acusaciones y responsable por la violencia sufrida.

Otro tipo de violencia es la sexual, la que no se presenta claramente evidenciada por las entrevistadas.

En un principio, el tema de la violencia se consideraba en el campo de los derechos humanos, hoy se ve cada vez más como un problema importante de salud pública, dada la frecuencia con que se presenta, así como la repercusión en los aspectos físico, psicológico de las víctimas, lo que ocasiona un vivir en sufrimiento, afectándose los diversos roles que cumplen las mujeres en la sociedad, el de madre fundamentalmente.

El contexto de la violencia

Respecto al contexto de la violencia, las razones y factores situacionales que llevaron al agresor a cometer la violencia, refieren las mujeres de este estudio, que cuestiones como el dinero y los celos tienden a ser el foco desencadenador de las discusiones, los que a su vez se muestran asociados al uso de alcohol y droga por el agresor, o todavía a la cultura machista de los hombres de considerarse con poder y pegar a las mujeres.

Refiere que los episodios de violencia son repetitivos y progresivamente más graves, caracterizándose por situaciones de cronicidad y creciente periodicidad. En el grupo investigado, constatamos tal consideración hecha por los autores, en la medida en que algunas mujeres refirieron el número de agresiones recibidas y un corto período de tiempo que estas ocurrían.

Los testimonios al respecto son:

...Perdida de 200 soles (E.1)

...Siempre bebe alcohol con su familia, con amigos...en la última discusión había fumado droga (E.2)

...Dice estar seguro que estoy saliendo con alguien...dice que me vió abrazada con un hombre (E.3).

El estudio ${ }^{(7)}$ de tipo cuantitativo, multicentrico y que entrevistó a 1,414 mujeres en Lima metropolitana y 1837 mujeres en el departamento del Cusco, cuyas edades oscilaron entre 15 a 49 años, fue constatado que tanto en
Lima metropolitana como en el departamento del Cusco, los celos y el estado de ebriedad del hombre son las situaciones más mencionadas. Resalta por su gran dimensión, también en el departamento del Cusco que el $63.5 \%$ de las mujeres mencionaron que su pareja se pone violento cuando está embriagado. También refirieron que un $19.1 \%$ de varones en Lima, se pone violento por problemas de dinero. Sin embargo, es importante mencionar que un $6.1 \%$ de las mujeres de Lima y un $8 \%$ en el Cusco manifiestan que no hay ninguna razón especial para que él se ponga violento.

La repercusión de la violencia sufrida por la mujer en su salud

En cuanto a la repercusión en la salud de la mujer, ciertas señales y síntomas manifestadas por las víctimas, tienden a no ser asociadas a la violencia sufrida, dificultando una evaluación efectiva de la magnitud del problema. Para el grupo de mujeres de estudio, la alteración fue mencionada por algunas de las entrevistadas. La violencia está asociada a mayores índices de suicidio, abuso de drogas, alcohol, quejas, cefaleas, disturbios gastrointestinales y sufrimiento psíquico en general. En el grupo identificamos en una de las entrevistadas, que estaba haciendo uso de sedantes; otras expresiones fueron:

...Casi no duermo (E.2)

...Cuando él me viene a buscar, me altera toda (son ex convivientes) (E.3)

Ramos (2001) enfatiza que aún cuando la violencia de género y el abuso de sustancias, son problemas asociados, no puede afirmarse que es una secuencia de causa-efecto, pues la violencia ocurre aún cuando no existe el consumo de drogas y afirma también que las mujeres que han experimentado violencia doméstica, tienen más riesgo de usar sustancias tóxicas. La especialista indicó que las mujeres que abusan del alcohol y otras drogas tienen más riesgo de ser víctimas de violencia doméstica y que los varones agresores, generalmente, son consumidores de sustancias tóxicas, pues la mayoría de ellos fueron criados por padres que abusaban de drogas o alcohol.

Por lo tanto, entorno a la violencia intrafamiliar, puede estar presente el uso de alcohol, drogas, medicamentos agravando la salud familiar. 
Reaccionando a la violencia sufrida a través de la denuncia

Las circunstancias en que viven las mujeres víctimas de violencia, muchas veces se sienten amenazadas e intimidadas, omitiendo el hecho y asumiendo sola las consecuencias del abuso sufrido. Buscamos identificar en las hablas de las entrevistadas que factores las llevaron a tomar la actitud de denunciar al compañero. Cuando lo hacen es por agotar todas las formas de soportar las agresiones o por razones de compromiso más profundo (agresor dirige la violencia a la hija).

...Estar harta...haber soportado por mucho tiempo (E.1)

...El miedo a que me vuelva a golpear (E.6)

...No puedo soportar más, parece una persona esquizofrénica, no creo que sea normal (E.4)

...Ha empezado a maltratar a mi hija y eso es muy grave (E.2)

Coherentemente con la construcción social de identidad femenina, en que la maternidad es un hecho fundamental de ser mujer, es que una de las entrevistadas tuvo el valor para denunciar. Según la cultura latina, sostiene Heilborn, las mujeres fueron naturalizadas en la condición de madre, diferente de la masculina, muchas veces construida en base a la fuerza física y la virilidad ${ }^{(9)}$.

El realizar la denuncia es sinónimo de "romper el silencio" y buscar ayuda en un servicio, principalmente en la policía cuando la situación es grave, temor a un asesinato o una posible violencia contra los hijos. La idea de mujer como ser pasivo tiende a ser considerada como justificativa de una actitud no reactiva ante el agresor, más no es apenas la ideología machista que se refleja en ese comportamiento, ha de considerarse la falta de soporte necesario a esas mujeres para que reviertan contra la situación que se encuentran, no se sienten seguras y amparadas para denunciar.

...Mi hijo mayor me defendió... fugamos a Lima para que mi hermano me ayude (E.1)

...Mi familia (madre, hermanos) no me ayudan, dicen que es mi problema (E.4)

...Unas amistades me aconsejaron de este lugar (Comisaría de Mujeres) que me podian orientar y ayudar (E.3).

Observa Franco, que las mujeres solo tienden a quejarse de la violencia cuando sienten apoyo de la red social que les garanticen solidaridad ante las consecuencias de tal decisión ${ }^{(10)}$.

Es en el medio familiar que la mujer tiende a buscar ayuda para liberarse de la situación de violencia en que se encuentra, entre tanto, eso no es una realidad para todas las mujeres, como es el caso de la entrevistada 4, conviviendo con la soledad de verse en una situación sin medios de solución. Ha de considerarse aún la ineficiencia de las estructuras sociales que no disponen de mecanismos efectivos de defensoría pública especial para las mujeres agredidas, puestos de salud con atención específica y, principalmente, protección, donde la integridad física de la mujer y de sus hijos, estaría resguardada por el poder público, de modo que permita a la mujer reestructurarse emocional y económicamente.

Otro factor que opera sobre las decisiones de las mujeres en denunciar, es la relación afectiva y de dependencia del compañero, que hace que estas no den visibilidad al carácter agresor, creyendo en cambios de comportamiento, es lo que se aprecia en los siguientes testimonios:

...Varias veces yo le perdone, él me decía que iba a cambiar, más no cumplió su palabra (E.1)

...Yo estaba esperando que él cambiase su forma de ser, más nunca cambió (E.3).

La experiencia social de las mujeres ante la violencia doméstica está integralmente condicionada por su posición social referida al género, en que la dominación masculina encuentra reunidas todas las condiciones de su pleno ejercicio, pues la primacía universal concedida a los hombres se afirma en la objetividad de las estructuras sociales $^{(11)}$.

Al respecto de las diferencias de género, Franco considera que las historias de violencia que alcanzan las mujeres y habitualmente las víctimas reciben consejos, que provienen del propio género (madre, vecina, amiga), para que evidencien la agresión recibida, no ejecutando la denuncia y soportando la situación. Tal situación coloca a la mujer en procesos constantes de ser re-víctima.

\section{CONSIDERACIONES FINALES}

- La violencia contra las mujeres se revela como importante tema social y sanitario, tanto del punto de vista de su magnitud como de las repercusiones en la morbilidad mortalidad de las mujeres, como de impacto social de la consecuencia en términos de calidad de vida.

- En el campo de la salud, área de nuestra atención es necesario reconocer a las mujeres en situación de 
violencia, lo que comprende considerar factores que las expone al riesgo de agresión, tales como el consumo de alcohol y drogas. Encontrándose con mayor frecuencia el uso de alcohol en los varones maltratadores, en menor proporción el uso de drogas; en el caso de la mujer, la situación de violencia, la coloca en mayor riesgo para el uso de sustancias tóxicas incluyendo fármacos. Se torna necesario continuar combatiendo el problema de violencia. - El profesional de salud debe ser capaz diagnosticar la violencia sexual, física, psicológica que muchas veces están encubiertas, expresadas por las usuarias a traves de quejas de dolores crónicos y sin causa aparente. Estar preparado para acoger las demandas de las mujeres prestando atención digna e integral.

- Es importante señalar que para que se tenga avances en combatir la violencia, es preciso trabajar en la bases que se refiere a la igualdad de géneros y en la concientización de los agravios a la salud producidos por el vicio de alcohol y de las drogas.

\section{AGRADECIMIENTOS}

Agradecemos a todos los docentes de la Escuela de Enfermería de Ribeirão Preto y a los treinta y tres participantes de nueve paises latinoamericanos que participaron en el proceso de implementación de los dos programas, y también al Gobierno del Japón y al Programa de Becas de la OEA por su apoyo financero y becas que possibilitaron la implementación del "I Programa Regional de Capacitación en Investigación para Enfermeros de América Latina".

\section{REFERENCIAS BIBLIOGRAFICAS}

1. Organización Mundial de la Salud. Dando prioridad a las mujeres: Recomendaciones éticas y de seguridad para la investigación sobre la violencia doméstica contra las mujeres. Red internacional de investigación sobre violencia contra las mujeres (Documento no oficial). Ginebra: OMS; 1999.

2. Salinas T. Familia, violencia y abuso de drogas. Violencia intrafamiliar y consumo de drogas. Bolivia: COPRE; 1999.

3. Castro A. Prevalencia de violencia intrafamiliar. PNUD contra la violencia, informe Perú. Lima; 2000.

4. Varcoe C. Abuse Obscure: An Ethnographic account of emergency nursing in relation to violence against women. Can J Nurs Res 2001; 32(4): 95-115.

5 . Koss MP. The negative impact of crime victimizations on women's survey. Violence against women. British Columbia, p. 5, 1998.

6. Minayo MC, Deslandes SF, Cruz O Neto, Gomes R. Pesquisa social. 19. ed. Petrópolis: Vozes; 1994.

7. Güezmes A. et al.Violencia Sexual y Física Contra la Mujeres. Estudio multicentrico de la OMS sobre la violencia de la pareja y la salud de las mujeres, Lima-Perú. Ginebra: Universidad Peruana Cayetano Heredia/OMS; 2002.

8. McFarlane J, Parker B, Soeken K, Berllock L. Assessing for abuse during pregnancy. Severity and frecuency of injuries and associated entry into prenatal care. JAMA 1992; 267(23): 3176-8.

9. Heilborn ML. Haciendo género. Una Antropología de la mujer en Brasil. In: Costa AO, Bruschini C, organizadores. Una cuestión de género. Sao Paulo (SP):Fundación Carlos Chagas/Rosa dos Ventos; 1992. p.93-128.

10. Franco IR. Mujeres en situación de violencia en el ámbito conyugal-las denunciasen la delegación de protección a la mujer de Salvador (BA). In: Barbosa MR; Aquino EML.

11. Bourdieu P. La dominación masculina. Rio de Janeiro (RJ): Bertrand Brasil, 1999. 